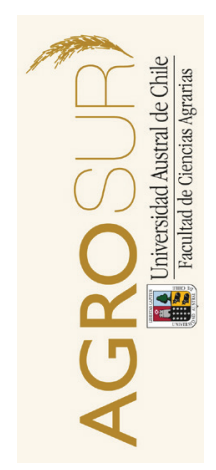

\title{
Caracterización de secuencias de los genes Ahas y psbA relacionados con resistencias a herbicidas en Lupinus albus $\mathrm{L}$.
}

\author{
Characterization of sequence of Ahas and $p s b A$ genes related \\ to herbicide resistance in Lupinus albus $\mathrm{L}$. \\ Vásquez, S. ${ }^{a, c}$, Mestanza, ${ }^{a, d}{ }^{a,}$ Riegel, . $^{b *}$ \\ ${ }^{a}$ Escuela de Graduados, Facultad de Ciencias Agrarias, Universidad Austral de Chile, Valdivia, Chile. \\ ${ }^{b}$ Instituto de Producción y Sanidad Vegetal, Facultad de Ciencias Agrarias, Universidad Austral de Chile, Valdivia, Chile. \\ ${ }^{c}$ Universidad Politécnica Salesiana, Cuenca, Ecuador. \\ ${ }^{d}$ Universidad Técnica Estatal de Quevedo, Quevedo, Ecuador.
}

\begin{tabular}{l} 
A R T I C L E IN F O \\
\hline Article history: \\
Received 02.10.2014 \\
Accepted 14.04.2015 \\
\hline Keywords: \\
Acetohydroxyacid synthase \\
acetolactate synthase \\
Ahas \\
Als \\
psbA \\
D1 \\
Lupinus albus \\
\hline
\end{tabular}

Original Research Article, Crop Science

*Corresponding author:

Santiago Vásquez, Ricardo Riegel

E-mail address:

rriegel@uach.cl

\begin{abstract}
A B S T R A C T
White lupine (Lupinus albus L.) is an important legume for human and animal consumption. However, this crop is a poor competitor with weeds, so their management is essential to increase yield. Among the herbicide most widely used for weed control in major crops, are inhibitors of acetohydroxyacid synthase (Ahas) (imidazolinone and sulfonylurea) and inhibitors of photosystem two (PII) (triazines, triazinones ureas). This study aimed to isolate and characterize Ahas and $p s b A$ genes related to resistance to herbicides inhibitor of Ahas and PII respectively. The coding region of these genes in Lupinus albus was amplified (PCR) and sequenced. These are potential sites containing the mutation involved in resistance to the described herbicides. In the gene Ahas we confirm the existence of a mutation ( $\mathrm{Gly}_{654} \rightarrow$ Asn) at a site previously reported to cause resistance to imidazolinones in other species. This point mutation is conserved in various legumes.
\end{abstract}

\section{RESUMEN}

El lupino blanco (Lupinus albus L.) es una importante leguminosa para la alimentación humana y animal. Sin embargo, este cultivo es un pobre competidor con las malezas, por lo que su manejo es fundamental para incrementar el rendimiento. Entre los herbicidas más utilizados para control de malezas en los principales cultivos están los inhibidores del acetohidroxiácido sintasa (Ahas) (imidazolinonas y sulfonilureas) y los inhibidores del fotosistema dos (PII) (triazinas, triazinonas, ureas). Este trabajo tuvo por objeto aislar y caracterizar los genes Ahas y $p s b A$ relacionados con la resistencia a herbicidas inhibidores de Ahas y PII respectivamente. Se amplificó (PCR) y secuenció la región codificante de Ahas y PsbA en Lupinus albus, los cuales están conteniendo los sitios cuya variación nucleotídica se asocia con la resistencia a estos herbicidas. En el gen Ahas se reporta la existencia de una mutación en un sitio previamente informado como causante de la resistencia a imidazolinonas $\left(\right.$ Gly $_{654} \rightarrow$ Asn), y se encuentra conservado en varias leguminosas.

Palabras clave: Acetohidroxiácido sintasa, Acetolactato sintasa, Ahas, Als, psbA, D1, Lupinus albus.

\section{INTRODUCCIÓN}

Lupinus es un género relativamente amplio y uno de los geográficamente más extensos, con una rica diversidad de especies (Gladstones, 1998). Las principales especies cultivadas a nivel mundial son: lupino de hoja angosta o australiano (Lupinus angustifolius L.), lupino blanco (Lupinus albus L.), lupino amarillo (Lupinus luteus L.), y lupino andino (Lupinus mutabilis Sweet.), las cuales presentan auto y polinización cruzada (Langridge y Goodman, 1985; Tay, 2009). Estas especies son consideradas de origen paleopoliploide (Atkins et al., 1998; Gladstones, 1998). En su temprana evolución probablemente ocurrieron eventos de auto y/o haloploidización y posteriormente un proceso de divergencia del genoma llevando a una "diploidización" (Wen- 
del, 2000). En la actualidad, las formas de lupino son funcionalmente diploides, sin embargo, los niveles de ploidía aún no están claros en muchas especies de este género (Naganowska et al., 2003). La domesticación de las especies cultivadas de lupino ocurrió paralelamente en regiones del Mediterráneo y los Andes, sin embargo, tienen una historia relativamente corta de domesticación en comparación con la mayoría de los cultivos (Cowling et al., 1998; Clements et al., 2005).

El grano de Lupinus albus es utilizado tanto para alimentación humana como animal debido a su alto contenido de proteína (Lagunes-Espinoza et al., 2000). Además, Lupinus albus tiene un bajo índice glucémico por lo que tiene importantes implicaciones para la salud humana (Phan et al., 2007). El lupino blanco es usado en la rotación de cultivos para reducir los agentes causantes de enfermedades, y para mantener la fertilidad del suelo a través de la fijación de nitrógeno atmosférico (Jones et al., 2003). Además, Lupinus albus se adapta bien a los suelos que tienen bajos niveles de fósforo debido a sus raíces proteiformes (Neumann y Martinoia, 2002).

El lupino blanco a pesar de ser un cultivo de follaje denso, es un mal competidor con las malezas debido a su lenta tasa de crecimiento en las primeras etapas, especialmente en el establecimiento del cultivo. Las malezas compiten con el lupino por el agua, nutrientes, luz solar y espacio, además pueden albergar plagas y patógenos causantes de enfermedades. Si no se realiza un adecuado control, las malezas pueden reducir el rendimiento significativamente. En Lupinus albus (Silva y Acevedo, 2003; Tay, 2009) y Lupinus angustifolius (Allen, 1977) informan que las malezas pueden ocasionar perdidas en el rendimiento de hasta el $70 \%$.

Dentro de un sistema de cultivo con mínima labran$\mathrm{za}$, los herbicidas se utilizan ampliamente para controlar las malezas. Así mismo, la tolerancia a los herbicidas amplía las opciones de manejo de malezas en un sistema productivo. Por ejemplo, en Australia, el manejo de las malezas ha sido fundamental para el desarrollo de Lupinus angustifolius como un cultivo exitoso, y los productos químicos han desempeñado un papel clave en este manejo (Bowran y Hashem, 2008), sin ellos es poco probable que este cultivo haya podido llegar a la superficie actual de 450.200 Ha (FAOSAT, 2014). Por lo tanto, un manejo adecuado de las malezas es fundamental para maximizar el rendimiento en lupino.

Las sulfonilureas (SU) e imidazolinonas (IMI) son familias de herbicidas de amplio uso. Estas se encuentran entre las opciones más populares para los agricultores de todo el mundo, porque no son tóxicos para los animales y son altamente selectivos (McCourt et al., 2006). Estos herbicidas son eficaces para matar plantas susceptibles mediante la inhibición de la acetohidroxiácido sintasa AHAS (EC 4.1.3.18), también conocida como acetolactato sintasa (ALS) (Duggleby y Pang
2000), que es una enzima clave en la biosíntesis de los aminoácidos de cadena ramificada tales como la valina, leucina e isoleucina en eucariotas y procariotas (McCourt et al., 2006). La inhibición de AHAS conduce a la muerte de la planta, principalmente debido a la inanición por falta de aminoácidos específicos, aunque otros efectos secundarios de la inhibición de AHAS también han sido implicados en el mecanismo de muerte de la planta (Shaner, 1991).

Otros grupos químicos como las triazinas, triazinonas, ureas y uracilos, también son herbicidas ampliamente utilizados para el control de malezas. Estos herbicidas a pesar de ser estructuralmente diversos tienen el mismo mecanismo de acción, inhiben la fotosíntesis de las plantas debido a que compiten con la plastoquinona (PQ) en el sitio de unión de la PQ con la proteína D1 dentro del complejo fotosistema dos (PSII). La inhibición del transporte de electrones del PSII detiene la producción de NADPH y ATP provocando una reducción del ciclo del carbono, lo que provoca una inanición de carbohidratos y estrés oxidativo (Powles y Yu, 2010). En Australia, donde el lupino de hoja angosta es la principal leguminosa cultivada, la metribuzina (triazinona) se usa ampliamente como herbicida post-emergente para el control de malezas dicotiledóneas (Perry et al., 1998; Bowran y Hashem 2008). Este tipo de herbicidas también se usa como pre-emergente para el control de malezas mono y dicotiledóneas en otros cultivos como soya (Glycine max L.) (Barrentine et al., 1982) y trigo (Triticum aestivum L.) (Kleemann y Gill, 2007).

Estudios sobre la resistencia a herbicidas en varios cultivos han encontrado diferentes modos de herencia y de acción genética. En diversas investigaciones ha sido posible inducir artificialmente mutaciones que otorgan resistencia a herbicidas inhibidores de AHAS. Estas estarían controladas por un gen semi-dominante en soya (Sebastian et al., 1989; Ghio et al., 2013), trigo (Newhouse et al., 1992) y raps (Brassica napus L.) (Swanson et al., 1989). En arroz (Oryza sativa L.) un gen estaría controlando la tolerancia a imidazolinona, mientras que en maíz (Zea mays L.) y raps dos genes con efecto aditivo estarían involucrados en la tolerancia a imidazolinona (Tan et al., 2005). Por otro lado, un solo gen dominante controla la tolerancia a metribuzina en genotipos de soya (Glycine max) (Edwards et al., 1976), y soya silvestre (Glycine soja Sieb. y Zucc.) (Kilen y He, 1992). Múltiples genes con efectos aditivos se han encontrado para la tolerancia metribuzina en los cultivares de trigo duro (Triticum durum Desf.) (Villarroya et al., 2000). En lupino de hoja angosta dos genes de naturaleza no alélica y con efecto aditivo estarían involucrado en la resistencia a metribuzina (Si et al., 2011), sin embargo, el mecanismo de tolerancia al herbicida no estaría basado en un sitio-objetivo, surgiendo que probablemente el metabolismo de la metribuzina está mediada por citocromo P450 (Pan et al., 2012). 
Para el lupino blanco a la fecha no hay descritas variantes nucleotídicas asociadas a la resistencia a herbicidas. Siendo las herramientas de control químico de malezas limitadas principalmente a herbicidas preemergentes.

El conocimiento del control genético, así como la ubicación y la secuencia de los genes implicados en la tolerancia a los herbicidas pueden ser de mucha utilidad para estrategias de mejoramiento genético en las que se combina el mejoramiento convencional y molecular. Por ejemplo, mediante la aproximación de la genética reversa usando la técnica de TILLING (Targeting Induced Local Lesions in Genomes) se inducen mutaciones y se identifican a nivel de ADN las plantas poseedoras de la mutación en el/los genes de interés. Este trabajo tuvo por objeto sentar las bases moleculares para una estrategia de este tipo, al identificar y caracterizar en Lupinus albus los genes Ahas y psbA asociados a resistencia a herbicidas.

\section{MATERIALES Y MÉTODOS}

\section{Material vegetal y aislamiento de ADN}

Semillas de Lupinus albus cv. Rumbo fueron sembradas en macetas y crecidas en invernadero, se cosecharon 5 hojas jóvenes de plantas individuales para el aislamiento del ADN. La extracción del ADN genómico se realizó utilizando bromuro de cetiltrimetilamonio (CTAB) (Almeyda et al., 1999) con adaptaciones realizadas por Muñoz (2011). La calidad y cantidad de ADN extraído fue evaluado por electroforesis en geles de agarosa al 1,5\%. El ADN fue cuantificado mediante espectrofotómetro Nanodrop midiendo la absorbancia a $260 \mathrm{~nm}$. El ADN fue diluido a una concentración final de $50 \mathrm{ng} / \mu \mathrm{l}$

\section{Amplificación PCR y secuenciación de los genes psbA y Ahas}

No existe información disponible en las bases de datos sobre las secuencias de los genes Ahas y psbA de Lupinus albus. En consecuencia, las secuencias de Ahas1 de soya (Glycine max) y fréjol (Phaseolus vulgaris L.) (Accesiones Genbank FJ581423.1 y GQ466185.1, respectivamente), y datos de secuenciación masiva de L. angustifolius (secuencia WGS) (GenBank: AOCW01146481.1) (Yang et al., 2013) se utilizaron para diseñar una serie de partidores para amplificar el gen Ahas. Mientras que para la amplificación del gen $p s b A$ se diseñaron partidores en base a la secuencia genómica del gen $p s b A$ de Lupinus luteus (NCBI: NC_023090.1) (Martin et al., 2014). El diseño de los partidores se realizó mediante el software Primer3 v.0.4 (http://bioinfo.ut.ee/primer3-0.4.0/) (Cuadro 1).

Para Ahas y psbA, la reacción PCR se llevó a cabo en un volumen de $50 \mu$ l que consistió en $2 \mu \mathrm{L}$ deADN, 36,6 $\mu \mathrm{L}$ de agua desionizada estéril, $5 \mu \mathrm{L}$ de Taq buffer $10 \mathrm{X}$ (Fermentas, Thermo Fisher Scientific Inc., USA), $1 \mu \mathrm{L}$ de dNTPs (10 mM) (Fermentas, Thermo Fisher Scientific Inc., USA), $3 \mu \mathrm{L}$ de $\mathrm{MgCl} 2$ (25 Mm) (Fermentas, Thermo Fisher Scientific Inc., USA), $1 \mu \mathrm{L}$ de cada partidor $(10 \mathrm{pmol} / \mu \mathrm{L})$ y $0,4 \mu \mathrm{L}$ de Taq polimerasa $(5 \mathrm{u} / \mu \mathrm{L})$ (Fermentas, Thermo Fisher Scientific Inc., USA). La PCR se realizó en un termociclador Labnet Multigene Gradient con el siguiente perfil: $94{ }^{\circ} \mathrm{C} 3 \mathrm{~min}, 37$ ciclos a 94 ${ }^{\circ} \mathrm{C} 45 \mathrm{~s}, 1 \mathrm{~min}$ a temperatura de alineamiento variable (Cuadro 1), y $72{ }^{\circ} \mathrm{C} 1 \mathrm{~min}, \mathrm{y}$ una extensión final de 7 min a $72{ }^{\circ} \mathrm{C}$. Los fragmentos de los genes $p s b A$ y Ahas fueron visualizados en geles de agarosa al 1,5\% teñidos con bromuro de etidio $(0,5 \mu \mathrm{g} / \mathrm{mL})$, los productos PCR fueron purificados y secuenciados directamente me-

Cuadro 1. Conjunto de partidores utilizados para la amplificación de los genes Ahas y PsbA.

Table 1. Primer sets used for Ahas and $p s b A$ genes amplification.

\begin{tabular}{|c|c|c|c|c|c|c|}
\hline Gen & Set & Nombre del partidor & & Secuencia $5^{\prime}-3^{\prime}$ & $\begin{array}{c}\text { Temperatura de } \\
\text { alineamiento }\left({ }^{\circ} \mathrm{C}\right)\end{array}$ & $\begin{array}{l}\text { Cobertura de } \\
\text { nucleótidos }^{\mathrm{a}}\end{array}$ \\
\hline \multirow[t]{6}{*}{ Ahas } & 1 & ALSLUP1F & $\mathrm{F}$ & TTCAGCGTCCTCCCTTCATC & 58 & 32 a 1058 \\
\hline & & ALSLUP2R & $\mathrm{R}$ & CCATGCATCCCAAGCATCTG & & \\
\hline & 2 & ALSLU10F & $\mathrm{F}$ & GGTACGGACGCTTTTCAAGA & 54 & 606 a 1429 \\
\hline & & ALSLU12R & $\mathrm{R}$ & CATTCGTCAGCTCATCCAAA & & \\
\hline & 3 & ALSLU9F & $\mathrm{F}$ & TGATTTTCGAGGTTGGAGAGA & 54 & 1305 a 1986 \\
\hline & & ALSLU11R & $\mathrm{R}$ & GGTTATCACGTCCTGGAAGG & & \\
\hline \multirow[t]{2}{*}{$p s b A$} & 4 & PSBLU6F & $\mathrm{F}$ & ATTCGTGTGCTTGGGAGTC & 56 & 0 a 1062 \\
\hline & & PSBLU9R & $\mathrm{R}$ & TGCTACTTTACTCTCAAAAGCTCGT & & \\
\hline
\end{tabular}

F: forward primer; R: reverse primer.

a La numeración de los nucleótidos se indican a partir del extremo 5' de la región codificante (CDS), en base a la secuencias de Ahas1 y psbA de Arabidopsis thaliana. 
diante método Sanger en un secuenciador capilar ABI 3730 xl (Macrogen Inc., Seúl, Korea).

\section{Análisis de secuencias}

Las secuencias fueron visualizadas mediante el software Chromas Lite 2.1.1. Para confirmar que las secuencias codifican los genes de interés, se comparó con secuencias existentes en la base de datos GenBank (http://www.ncbi.nlm.nih.gov/genbank/) utilizando el programa de búsqueda Basic Local Alignment Search (BLAST) (http://blast.ncbi.nlm.nih.gov/Blast.cgi). Se realizaron alineamientos usando el programa Clustal Omega (http://www.ebi.ac.uk/Tools/msa/clustalo/) con secuencias codificantes de los genes $p s b A$ y Ahas de otras leguminosas existentes en la base de datos GenBank. Las secuencias de aminoácidos fueron predichas mediante el software Orf-Finder (http://www.ncbi. nlm.nih.gov/projects/gorf/).

\section{RESULTADOS Y DISCUSIÓN}

\section{Análisis del gen Ahas}

El set de partidores permitieron amplificar la región codificante (CDS) parcial del gen Ahas de Lupinus albus, el fragmento corresponde a $1651 \mathrm{pb}$ de nucleótidos. Ahas de L. albus mostró alineamiento significativo con el gen Ahas1 de Cicer arietinum L. 84\% (XM_004485696.1); Ahas2 de Glycine max 84\% (XM_003543011.2); Ahas de Phaseolus vulgaris 83\% (KF378787.1). Además, se realizó un análisis Blast con datos de secuenciación masiva del genoma de $\mathrm{Lu}$ pinus angustifolius (whole genome shotgun sequence) (Yang et al., 2013), este análisis mostró alineamiento significativo sólo con dos secuencias (GenBank: AOCW01126472.1 y AOCW01146481.1) 96 y $89 \%$ respectivamente, estos resultados sugieren que son genes homólogos de Ahas en Lupinus angustifolius.

La región del gen Ahas en Lupinus albus no está interrumpida por intrones, lo que está de acuerdo con los genes Ahas reportados. Ésta es característica de casi todas las plantas superiores en las que se ha estudiado este gen, hasta la actualidad en sólo tres especies vegetales se han reportado intrones, en Lindernia spp, (Uchino y Watanabe, 2002), Schoenoplectus juncoides Roxb. (Uchino et al., 2007) y Schoenoplectus mucronatus L. (Scarabel et al., 2010).

Algunos productos secuenciados se descartaron por la presencia de pics solapados en el cromatograma, lo que probablemente sugiere la presencia de más de una copia de Ahas en L. albus; de hecho las plantas superiores tienen un número variable de genes Ahas, lo que depende principalmente del nivel de ploidía (Azevedo et al., 2006). Por ejemplo Arabidospsis thaliana L. (diploide) tiene una copia constitutiva, Nicotiana tabacum L. (alotetraploide) tiene dos loci disociados de Ahas, cuyos genes tienen expresión coordinada en todos los órganos (Mazur et al., 1987); Zea mays, considerado un alopoliploide críptico, tiene dos genes Ahas muy similares; Brassica napus, un anfidiploide de Brassica campestris L. y Brassica oleracea L., tiene cinco genes Ahas, las diferentes isoformas son reguladas en el desarrollo de tejidos específicos (Singh, 1999). Estudios recientes en Glycine max (fabaceae) (Ghio et al., 2013), encontraron cuatro copias del gen Ahas, sin embargo solo una (Als1) sería la que más se expresa en las hojas y estaría controlando la resistencia a SU. Mientras que Walter et al. (2014) aislaron cinco genes Ahas y dos de estos (Als1 y Als2) estarían involucrados en el control de la resistencia a SU en soya.

Los antecedentes de la literatura y los resultados de secuenciación, sugieren que probablemente existe más de una copia del gen Ahas en Lupinus albus, lo que abre nuevas perspectivas en el control genético de la resistencia a herbicidas útiles para el mejoramiento genético de este cultivo. De confirmarse la presencia de más copias del gen Ahas se requiere profundizar en su estudio para determinar el papel que jugarían en el control de la resistencia a herbicidas inhibidores de la enzima AHAS.

A nivel de aminoácidos AHAS de Lupinus albus, predijo una secuencia (parcial) de 550 aa lo que está de acuerdo con la mayoría de plantas en donde estos genes se componen de aproximadamente 660 aa variando entre las especies (Pang et al., 2002). La secuencia de L. albus en aminoácidos exhibe identidad significativa con AHAS1 de Cicer arietinum 89\% (XP_004485753.1); AHAS2 de Glycine max 89\% (XP_003543059.1); AHAS de Ricinus communis L. 90\% (XP_002511176.1) y AHAS de Phaseolus vulgaris 88\% (ACV84152.1, resultados que confirman que se trata del gen Ahas.

Estudios de AHAS indican que presenta cinco dominios discretos (A, B, C, D y E), cada dominio comprendido entre 4 y 19 aa (12 a $57 \mathrm{pb}$ ) (Boutsalis et al., 1999). Los dominios C, A y D abarcan los residuos 115 a 133, 191 a 203 y 205 a 210 del gen Ahas respectivamente; el dominio B comprende los residuos 573 a 576 y el dominio E abarca los residuos 651 a 655 (Park y Mallory-Smith, 2004). Las mutaciones que se producen en posiciones aminoacídicas específicas dentro de cada uno de estos dominios resultan en una reducción de la sensibilidad a ciertas clases de inhibidores de Ahas (Tan et al., 2005).

La región amplificada en Lupinus albus está conteniendo todos los sitios cuya variación nucleotídica se asocia a la resistencia a herbicidas inhibidores de AHAS descritas hasta ahora, tanto en malezas como en cultivos (Figura 1). Las mutaciones en las posiciones Ala ${ }_{122} \rightarrow$ Thr y Tyr, Ala ${ }_{205} \rightarrow$ Val, Ser $_{653} \rightarrow$ Thr, Asn, Ile y $\mathrm{Gly}_{654} \rightarrow$ Glu y Asp (de acuerdo con la numeración adoptada para la secuencia de Ahas de Arabidopsis thaliana) provocan resistencia a los herbicidas IMI; particularmente la mutación en $\operatorname{Ser}_{653}$ que confiere tolerancia a 


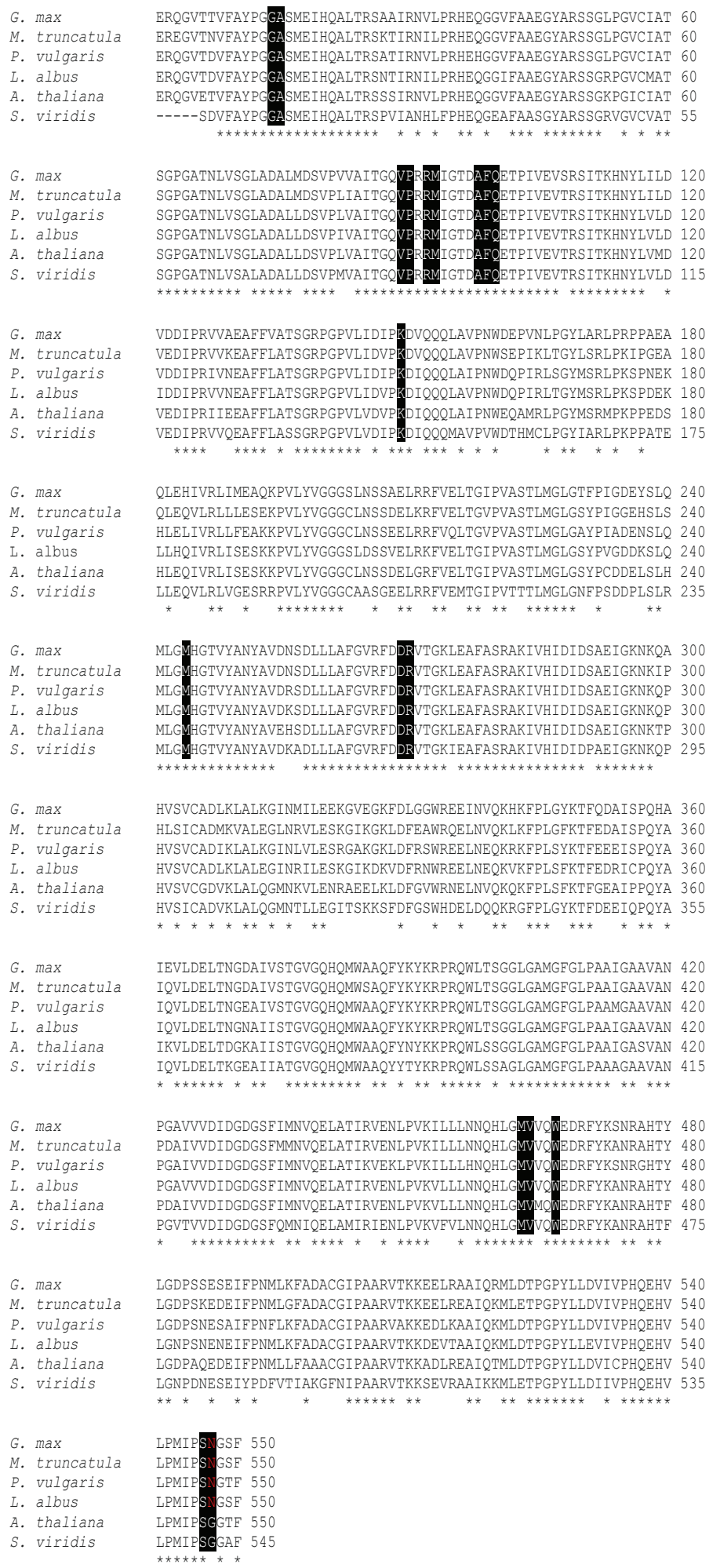

Figura 1. Comparación de secuencias de aminoácidos de Ahas1 entre diferentes especies. Los sitios en negro indican las mutaciones reportadas que causarían resistencia a herbicidas inhibidores de Ahas. Los aminoácidos en rojo indican la mutación encontrada en Lupinus albus y otras leguminosas ( $\mathrm{Gly}_{654} \rightarrow$ Asn). Accesiones Genbank: Arabidopsis thaliana (NP_190425.1); Phaseolus vulgaris ACV84152.1; Setaria viridis (AGU13757.1); Glycine max (AGL09062.1) Medicago truncatula (AES73057).

Figure 1. Comparison of Ahas1 amino acid sequences between different species. The sites in black indicate the reported mutations cause resistance to Ahas inhibiting herbicides. Amino acids in red indicate the mutation found in Lupinus albus and other legumes (Gly ${ }_{654} \rightarrow$ Asn). Genbank accessions: Arabidopsis thaliana (NP_190425.1); Phaseolus vulgaris ACV84152.1; Setaria viridis (AGU13757.1); Glycine max (AGL09062.1) Medicago truncatula (AES73057). 
IMI, pero no tolerancia cruzada a otros inhibidores de Ahas, ha sido utilizada para el desarrollo de cultivos tolerantes a IMI (Sathasivan et al., 1991; Dietrich, 1998; Lee et al., 1999; Tranel y Wright, 2002). Las mutaciones

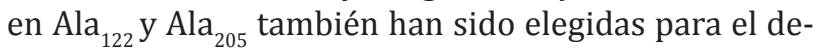
sarrollo de cultivos tolerantes a IMI (Bright et al., 1992; Tranel y Wright, 2002; White et al., 2003). Las mutaciones en Pro $_{197}$ están asociadas con la resistencia sólo a los herbicidas SU, con la excepción de Leu, que también provoca la resistencia a IMI (Powles y Yu, 2010).

Mutaciones en la posición Asp $_{376} \rightarrow$ Glu y $\operatorname{Trp}_{574} \rightarrow$ Leu, Arg confieren resistencia a todas las clases de inhibidores de Ahas en diferentes grados, mientras que una sustitución en $\mathrm{Arg}_{377} \rightarrow$ His conferiría la resistencia a SU, SCT (sulfonilaminocarboniltriazolinonas) y TP (triazolopyrimidinas) (Massa et al., 2011). La mutación en la $\operatorname{Trp}_{574}$ provoca tolerancia cruzada a diferentes familias de herbicidas que inhiben Ahas y ha sido utilizado para el desarrollo de cultivos tolerantes a IMI (Bernasconi et al., 1995; Tranel y Wright, 2002). La mayoría de cultivos comercializados como tolerantes a IMI han sido desarrollados ya sea por una mutación puntual o una combinación de $\mathrm{Ala}_{205}, \operatorname{Trp}_{574}$ y $\operatorname{Ser}_{653}$ (Bernasconi et al., 1995; Dietrich, 1998; Croughan, 2002; Tan et al., 2005).

El análisis a nivel de aminoácidos de Lupinus albus presenta una mutación en el aminoácido $\mathrm{Gly}_{654} \rightarrow$ Asn (Figura 1). Una mutación en el aminoácido Gly ${ }_{654} \rightarrow$ Asp ha sido reportado como un sitio que confiere resistencia a IMI en Setaria viridis L. (Poaceae) (Laplante et al., 2009). Sin embargo, AHAS de Lupinus albus al comparar con AHAS1 de otras leguminosas como: Medicago truncatula L., Glycine max, Phaseolus vulgaris, se observa que estas especies también presentan la mutación Gly $_{654} \rightarrow$ Asn, lo que parece ser una característica propia de varias leguminosas. De hecho, para determinadas zonas de EEUU, el herbicida Imazethapyr (IMI) (PursuitFASF) (Ihibidor de AHAS), es comercializado para su uso pre y post-emergente, en alfalfa, trébol y varias leguminosas comestibles incluyendo maní, frejol, arveja y soya.

Para Lupinus albus, desde la decada del 90, hay datos promisorios para el uso de imazethapyr (IMI) como herbicida selectivo postemergencia (Yates, 1993). Sin embargo, como demuestran Pooran et al. (2013) en Cicer arietinum, el nivel de resistencia a imazethapyr dentro de la misma especie puede presenter gran variabilidad. Descrita la secuencia del gen Ahas, se pueden aplicar estrategias moleculares como el EcoTILLING, que usa las técnicas del TILLING para identificar mutaciones naturales en plantas individuales dentro de un banco de germoplasma o poblaciones naturales (Comai et al., 2004; Nieto et al., 2007).

\section{Análisis del gen $p s b A$}

El set de partidores amplificaron la región codificante (CDS) completa del gen $p s b A(1062 \mathrm{pb})$ de Lupinus al- bus, esta región mostró $99 \%$ de identidad con el gen $p s b A$ de Lupinus luteus (GenBank: KC695666.1) (Martin et al., 2014), y 97\% con los genes $p s b \mathrm{~A}$ de otras leguminosas como: Lens culinaris Medik. (KF201590.1), Glycyrrhiza glabra L. (KF186232.1) y Vicia faba L. (KF042344.1).

En el análisis a nivel de aminoácidos, $p s b A$ está codificando la proteína D1 que tiene 353 aa. La proteína D1 de Lupinus albus mostró 99\% de identidad con D1 de Lupinus luteus (YP_008963581.1); Phaseolus vulgaris (YP_001122788.2), Glycine max (YP_538745.1); Lotus japonicus L. (NP_084778.1) y Cicer arietinum (YP_002149715.1). Se observa que el gen $p s b A$ está altamente conservado entre las leguminosas, presentado la misma longitud y alta similitud entre las secuencias. De hecho, se conoce que la mayoría de las angiospermas fotosintéticas tienen una organización, estructura y contenido génico del cloroplasto altamente conservado (Clegg et al., 1994; Timmis et al., 2004; Landau, 2008; Jansen y Ruhlman, 2012).

Por lo tanto, estos resultados sugieren que el gen amplificado corresponde al gen $p s b A$ de Lupinus albus. El alineamiento entre las secuencias codificantes de psbA de Lupinus albus y Lupinus luteus revelan una mutación de un nucleótido, $\mathrm{C}_{1037} \rightarrow \mathrm{T}$ que está modificando el aminoácido $\mathrm{Ala}_{346} \rightarrow$ Val en la proteína D1(Figura 2).

Una de las características más relevantes de la resistencia a la triazina (inhibidor del PSII), es que una mutación única estaría controlando el mecanismo de resistencia. Esta mutación puntual se encuentra en el gen $p s b A$ cloroplastídico de herencia materna y provoca una sustitución de aminoácidos $\operatorname{Ser}_{264} \rightarrow$ Gly en el sitio de unión de la PQ (Goloubinoff et al., 1984; Hirschberg y McIntosh., 1983). Con algunas excepciones, prácticamente todas las especies de malezas resistentes a triazina tienen esta mutación (Powles y Yu, 2010). Sin embargo, los herbicidas PSII no triazinas tienen una actividad normal debido a que tienen una distinta unión química, es decir los herbicidas no triazinas a pesar de ser inhibidores del PSII, actuarían normalmente en plantas resistentes a las triazinas. Por lo tanto, la resistencia a los herbicidas PSII no triazinas requieren de diferentes mutaciones, y de hecho cinco de estas mutaciones se han reportado en algunas malezas. En Portulaca oleracea L. seleccionada por resistencia a herbicidas inhibidores del PII como triazina y urea, muestran una mutación $\operatorname{Ser}_{264} \rightarrow$ Thr en la proteína D1 que confiere resistencia a ambos herbicidas (Masabni y Zandstra, 1999). Una mutación puntual Val $_{219} \rightarrow$ Ile en dos malezas, Kochia scoparia L. y Poa annua L. provoca resistencia a metribuzina (triazinona) y diuron (urea) (Mengistu et al., 2000; Mengistu et al., 2005). La mutación $\mathrm{Asn}_{266} \rightarrow$ Thr en la maleza Senecio vulgaris L. provoca resistencia a bromoxynil y terbacil (Park y MallorySmith, 2006). La mutación $\mathrm{Ala}_{251} \rightarrow$ Val en Chenopodium álbum L. provoca resistencia a metamitron (metamitrona) (Mechant et al., 2008) y finalmente la mutación 


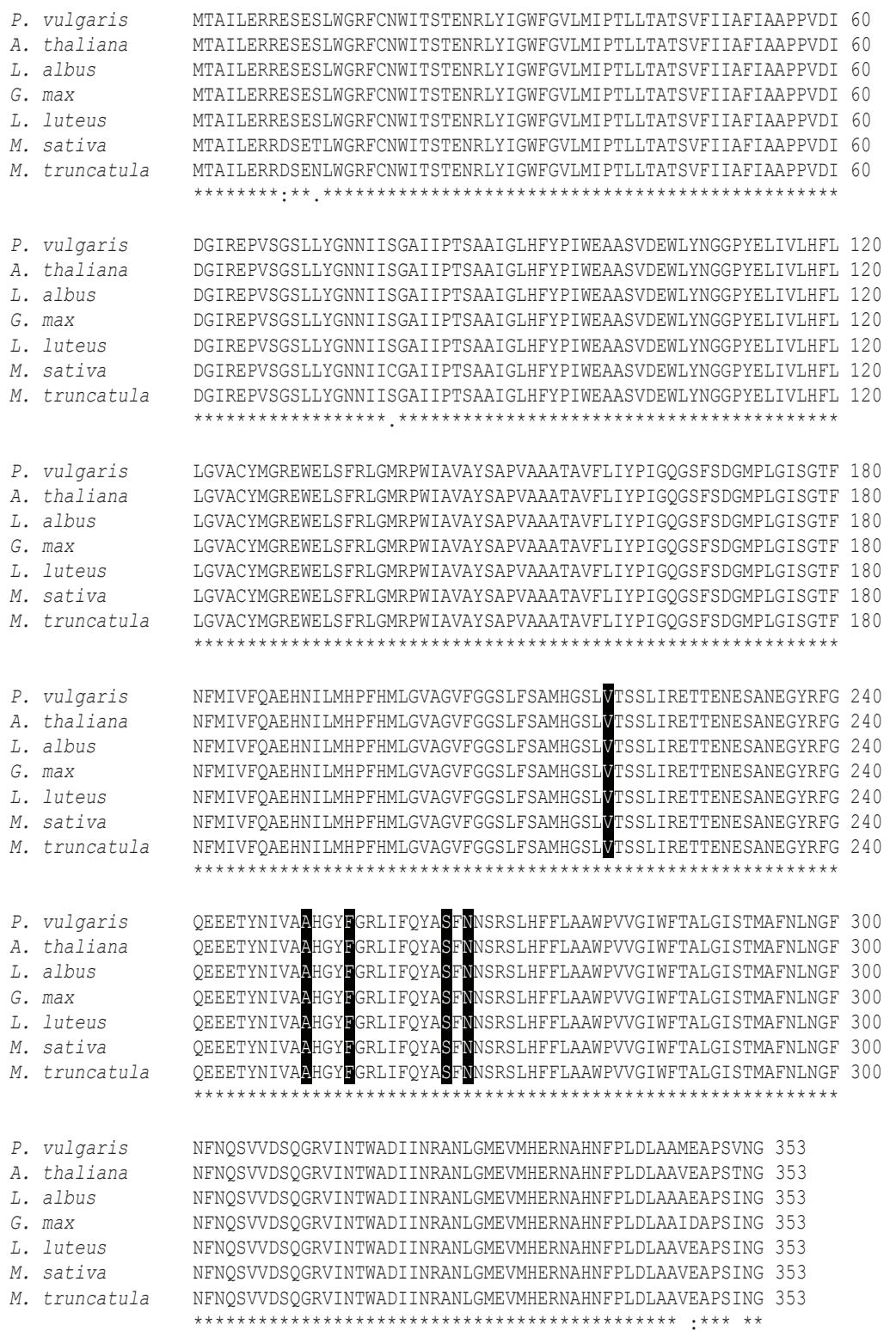

Figura 2. Comparación de secuencias de aminoácidos de la Proteína D1 entre diferentes especies. Los sitios en negro indican las mutaciones que causarían resistencia a herbicidas inhibidores de PSII. Accesiones Genbank: Arabidopsis thaliana: (NP_051039.1); Medicago truncatula: (AGV52623.1); Phaseolus vulgaris: (Q01366.2); Lupinus luteus: (YP_008963581.1); Medicago sativa (P04998.1); Glycine max: (YP_538745.1).

Figure 2. Comparison of amino acid sequences of the D1 protein from different species. The sites in black indicate the mutations cause resistance to PSII inhibiting herbicides. Genbank accessions: Arabidopsis thaliana: (NP_051039.1); Medicago truncatula: (AGV52623.1); Phaseolus vulgaris: (Q01366.2); Lupinus luteus: (YP_008963581.1); Medicago sativa (P04998.1); Glycine max: (YP_538745.1).

$\mathrm{Phe}_{255} \rightarrow$ Ile en la maleza Capsella bursa-pastoris L. provoca resistencia a triazinonas (Perez-Jones et al., 2009).

La región amplificada del gen $p s b A$ de Lupinus albus está conteniendo todos los sitios cuya mutación potencial es asociada con la resistencia a herbicidas. Por lo tanto, esta región contendría importante información del control genético de la resistencia a herbicidas que inhiben el PSII.

\section{CONCLUSIONES}

Se logró aislar y amplificar una región parcial del gen Ahas y la región codificante completa del gen $p s b A$ de L. albus, los cuales están conteniendo todos los sitios descritos hasta ahora cuya variación nucleotídica comúnmente se asocia a la resistencia a herbicidas inhibidores de AHAS y PII respectivamente. 
La información generada para esta especie permite complementar a futuro estrategias de mejoramiento convencional con herramientas moleculares como la genética reversa.

Se confirma la existencia de una mutación en un sitio previamente reportado como causante de la resistencia a imazethapyr $\left(\mathrm{Gly}_{654} \rightarrow\right.$ Asn) y está conservado en varias leguminosas.

\section{Agradecimientos.}

Agradecemos especialmente a Judith Carrasco P., y al personal del Laboratorio de Biotecnología Silvoagrícola por la asistencia técnica.

\section{REFERENCIAS}

Allen, J., 1977. Weeds in grain-lupins. 1. The effect of weeds on grain-lupin yields. Australian Journal of Experimental Agriculture and Animal Husbandry 17, 112-117.

Almeyda, L., Rubio, O., Cadena, H., Díaz, M., Zavala, T., Rocha, P., Díaz, A., 1999. Implementación de técnicas moleculares para la detección del agente causal de la punta morada de la papa en plantas e insectos vectores. Proyecto de investigación, Laboratorio de Patología MolecularMonterrey. INIFAP, Informe de Avances. 19 p.

Atkins, C., Smith, P., Gupta, S., Jones, M., Caligari, P., 1998. Genetics, cytology and biotechnology, in: Gladstones, J., Atkins, C., Hamblin, J. (Eds.), Lupins as crop plants: biology, production and utilization. CAB International, London, pp. 67-92.

Azevedo, R., Lancien, M., Lea, P., 2006. The aspartic acid metabolic pathway, an exciting and essential pathway in plants. Amino Acids 30, 143-162.

Barrentine, W., Hartwig, E., Edwards, C., Kilen T., 1982. Tolerance of 3 soybean (Glycine max) cultivars to metribuzin. Weed Science 30, 344-348.

BASF Corporation. 2011. Pursuit Herbicide. 26 Davis Drive, Research Triangle Park, NC 27709. 27p.

Bernasconi, P., Woodworth, A., Rosen, B., Subramanian, M., Siehl, D., 1995. A naturally occurring point mutation confers broad range tolerance to herbicides that target acetolactate synthase. The Journal of Biological Chemistry 270, 17381-17385.

Boutsalis, P., Karotam, J., Powles, S., 1999. Molecular basis of resistance to acetolactate synthase-inhibiting herbicides in Sisymbrium orientale and Brassica tournefortii. Pest Management Science. 55(5), 507-516.

Bowran, D., Hashem, A., 2008. The role of weed management in sustaining systems for lupin production, in: Palta, J.A., Berger, J.D. (Eds.), Proceedings of the $12^{\text {th }}$ International Lupin Conference, Fremantle, Australia, 14-18 September, pp. 11-14.

Bright, S., Chang, M., Evans, I., Macdonald, M., (1992). Herbicide resistant plants. Patent Application of World Intellectual Property Organization W092/08794.

Clegg, M.T., Gaut, B.S., Learn, G.H., Morton, B.R., 1994. Rates and patterns of chloroplast DNA evolution. Proceedings of the National Academy of Sciences of the United States of America 91(15), 6795-6801.

Clements, J., Dracup, M., Buirchell, B., Smith, C., 2005. Variation for seed coat and pod wall percentage and other traits in a germplasm collection and historical cultivars of lupins. Australian Journal of Agricultural Research 56, 75-83.

Comai, L., Young, K., Till, B.J., Reynolds, S.H., Greene, E.A., Codomo, C., Enns, L., Johnson, J., Burtner, C., Odden, A., Henikoff, S., 2004. Efficient discovery of DNA polymorphisms in natural populations by Ecotilling. The Plant Journal 37(5), 778-786.

Cowling, W., Huyghe, C., Swiecicki, W., 1998. Lupin breeding, in: Gladstones, J.S., Atkins, C.A., Hamblin, J., (Eds.), Lupins as crop plants: biology, production and utilization. CAB International, London, pp. 93-120.

Croughan, T., (2002). Herbicide resistant rice, US Patent Application 20020019313.

Dietrich, G., (1998). Imidazolinone resistant AHAS mutants, US Patent 5767361.

Duggleby, R., Pang, S., 2000. Acetohydroxyacid synthase. Journal of biochemistry and molecular biology 33, 1-36.

Edwards, C., Barrentine, W., Kilen, T., 1976. Inheritance of sensitivity to metribuzin in soybeans. Crop Science 16, 119-120.

Food and Agriculture Organization of the United Nations (FAOSTAT), http://faostat.fao.org/ (acceso 01.12.2014).

Ghio, C., Ramos, M., Altieri, E., Bulos, M., Sala, C., 2013. Molecular characterization of Als1, an acetohydroxyacid synthase mutation conferring resistance to sulfonylurea herbicides in soybean. Theoretical and Applied Genetics. 126, 2957-2968.

Gladstones, J., 1998. Distribution, origin, taxonomy, history and importance, in: Gladstones, J., Atkins, C., Hamblin, J. (Eds.), Lupins as crop plants. Biology: production and utilization. CAB International, Oxon, pp. 1-39.

Goloubinoff, P., Edelman, M., Hallick, R., 1984. Chloroplast-encoded atrazine resistance in Solanum nigrum: $p s b A$ loci from susceptible and resistant biotypes are isogenic except for a single codon change. Nucleic Acids Research 12, 9489-96.

Hirschberg, J., McIntosh, L., 1983. Molecular basis of herbicide resistance in Amaranthus hybridus. Science 222, 1346-49.

Jansen, R., Ruhlman, T., 2012. Plastid genomes of seed plants, in: Bock, R., Knoop, V. (Eds.), Genomics of chloroplast and mitochondria. New York, Springer, pp. 103-126.

Jones, R., Coutts, B., Cheng, Y., 2003. Yield limiting potential of necrotic and non-necrotic strains of Bean yellow mosaic virus in narrow-leafed lupin (Lupinus angustifolius). Australian Journal of Agricultural Research 54, 849-859.

Kilen, T., He, G., 1992. Identification and inheritance of metribuzin tolerance in wild soybean. Crop Science 32, 684-685.

Kleemann, S., Gill, G., 2007. Differential tolerance in wheat (Triticum aestivum L.) genotypes to metribuzin. Australian Journal of Agricultural Research 58, 452-456.

Lagunes-Espinoza, L., Huyghe, C., Papineau, J., 2000. Genetic variation for pod wall concentration in Lupinus albus. Plant Breeding 119, 421-425.

Landau, A.M., 2008. Análisis molecular y funcional de líneas citoplásmicas con deficiencias clorofílicas originadas 
por un gen mutador de cloroplastos de cebada. Tesis Doctorado Ciencias Biológicas, Universidad de Buenos Aires. 116 p.

Langridge, D., Goodman, R., 1985. Honeybee pollination of lupins (Lupinus albus cv. Hamburg). Australian Journal of Agricultural Research 25, 220-223.

Laplante, J., Rajcan, I., Tardif, F., 2009. Multiple allelic forms of acetohydroxyacid synthase are responsible for herbicide resistance in Setaria viridis. Theoretical and Applied Genetics 119, 577-585.

Lee, Y., Chang, A., Duggleby, R., 1999. Effect of mutagenesis at serine 653 of Arabidopsis thaliana acetohydroxyacid synthase on the sensitivity to imidazolinone and sulfonylurea herbicides. FEBS Letters 452, 341-345.

Martin, G.E., Rousseau-Gueutin, M., Cordonnier, S., Lima, O., Michon-Coudouel, S., Naquin, D., Ferreira De Carvalho, J., Aïnouche, M., Salmon, A., Aïnouche, A., 2014. The first complete chloroplast genome of the Genistoid legume Lupinus luteus: evidence for a novel major lineage-specific rearrangement and new insights regarding plastome evolution in the legume family. Annals of botany 113(7), 1197-1210.

Massa, D., 2011. Investigations on herbicide resistance in Apera spicaventi populations. PhD Thesis. University of Hohenheim, Germany. 87p.

Masabni, J., Zandstra, B., 1999. A serine-to-threonine mutation in linuron-resistant Portulaca oleracea. Weed Science 47, 393-400.

Mazur, B., Chui, C., Smith, J., 1987. Isolation and characterization of plant genes coding for acetolactate synthase, the target enzyme for two classes of herbicides. Plant physiology 85, 1110-1117.

McCourt, J., Pang, S., King-Scott, J., Guddat, L., Duggleby, R., 2006. Herbicide-binding sites revealed in the structure of plant acetohydroxyacid synthase, Proceedings of the National Academy of Sciences 103, 569-573.

Mechant, E., De Marez, T., Hermann, O., Olsson, R., Bulcke, R., 2008. Target site resistance to metamitron in Chenopodium album L. Journal of Plant Diseases and Protection 21, 37-40.

Mengistu, L., Mueller-Warrant, G., Liston, A., Barker, R., 2000. psbA mutation (valine219 to isoleucine) in Poa annua resistant to metribuzin and diuron. Pest Management Science 56, 209-17.

Mengistu, L., Christoffers, M., Lym, R., 2005. A psbA mutation in Kochia scoparia (L) Schrad from railroad rightsof-way with resistance to diuron, tebuthiuron and metribuzin. Pest Management Science 61, 1035-42.

Miflin, M., 1974. The location of nitrite reductase and other enzymes related to amino acid biosynthesis in the plastids of root and leaves. Plant Physiology 54, 550-555.

Muñoz, M., 2011. Caracterización de genes involucrados en la biosíntesis de isoflavonoides y su asociación con la resistencia a antracnosis en Lupinus albus L. Tesis Doctorado Ciencias Agrarias, Universidad Austral de Chile. $134 \mathrm{p}$.

Naganowska, B., Wolko, B., Sliwińska, E., Kaczmarek, Z., 2003. Nuclear DNA content variation and species relationships in the genus Lupinus (Fabaceae). Annals of Botany 92, 349-355.

Neumann, G., Martinoia, E., 2002. Cluster roots an under- ground adaptation for survival in extreme environments. Trends in Plant Science. 7, 162-167.

Newhouse, K., Smith, W., Starrett, M., Schaefer, T., Singh, B., 1992. Tolerance to imidazolinone herbicides in wheat. Plant Physiology 100, 882-886.

Nieto, C., Piron, F., Dalmais, M., Marco, C.F., Moriones, E., Gómez-Guillamón, M.L., Truniger, V., Gómez, P., Garcia-Mas, J., Aranda, M., Bendahmane, A., 2007. EcoTILLING for the identification of allelic variants of melon eIF4E, a factor that controls virus susceptibility. BMC Plant Biology 7, 1-34.

Pan, G., Si, P., Yu, Q., Tu, J., Powles, S., 2012. Non-target site mechanism of metribuzin tolerance in induced tolerant mutants of narrow-leafed lupin (Lupinus angustifolius). Crop \& Pasture Science 63, 452-458.

Pang, S., Duggleby, R., Guddat, L., 2002. Crystal structure of yeast acetohydroxyacid synthase: a target for herbicidal inhibitors. Journal of Molecular Biology 317, 249-262.

Park, K., Mallory-Smith, C., 2004. Physiological and molecular basis for ALS inhibitor resistance in Bromus tectorum biotypes. Weed Research 44, 71-77.

Park, K., Mallory-Smith, C., 2006. psbA mutation (Asn266 to Thr) in Senecio vulgaris L. confers resistance to several PSII-inhibiting herbicides. Pest Management Science 62, 880-85.

Perez-Jones, A., Intanon, S., Mallory-Smith, C., 2009. psbA mutation (Phe 255 to Ile) in Capsella bursa-pastoris confers resistance to triazinone herbicides. Weed Science 57, 574-78.

Phan, H., Ellwood, S., Adhikari, K., Nelson, M., Oliver, R., 2007. The first genetic and comparative map of white lupin (Lupinus albus L.): identification of QTLs for anthracnose resistance and flowering time and a locus for alkaloid content. DNA Research 14, 59-70.

Perry, M., Dracup, M., Nelson, P., Jarvis, R., Rowland, I., French, R., 1998. Agronomy and farming systems, in: Gladstones, J., Atkins, C., Hamblin, J. (Eds.), Lupins as crop plants, biology, production and utilization. CAB International, Wallingford, UK, pp. 291-338.

Pooran, G., Aravind, J., Srinivasan, S., Sushil, C., Sarvjeet, S., Shailesh, T., Inderjit, S., Guriqbal, S., Tapas, D., Muraleedhar, A., Neelu, M., Nagasamy, N., Laxmipathi, G., 2013. Large genetic variability in chickpea for tolerance to herbicides imazethapyr and metribuzin. Agronomy 3, 524-536.

Powles, S., Yu, Q., 2010. Evolution in Action: Plants resistant to herbicides. Annual Review of Plant Biology 61, 317-347.

Sathasivan, K., Haughn, G., Murai, N., 1991. Molecular basis of imidazolinone herbicide resistance in Arabidopsis thaliana var Columbia. Plant Physiology 97, 1044-1050.

Scarabel, L., Locascio, A., Furini, A., Sattin, M., Varotto, S., 2010. Characterisation of ALS genes in the polyploid species Schoenoplectus mucronatus and implications for resistance management. Pest Management Science 66(3), 337-44.

Singh, B., 1999. Plant amino acids: biochemistry and biotechnology. Marcel Dekker, New York.

Shaner, D., 1991. Physiological effects of the imidazolinone herbicides, in: Shaner, D., O'Connor S. (Eds.), The Imidazolinone Herbicides. CRC Press, Boca Raton, pp. 129138. 
Sebastian, S., Fader, G., Ulrich, J., Forney, D., Chaleff, R., 1989. Semidominant soybean mutation for resistance to sulfonylurea herbicides. Crop Science 29, 1403-1408.

Si, P., Pan, G., Sweetingham, M., 2011. Semi-dominant genes confer additive tolerance to metribuzin in narrow-leafed lupin (Lupinus angustifolius L.) mutants. Euphytica 177, 411-418.

Silva, P., Acevedo, E., 2003. Cultivo de lupino en la precordillera de la VIII Región. Boletín divulgativo №1. FONDEF D99I1081, Santiago, Chile, pp. 1-6. http://www. sap.uchile.cl/descargas/lupino/Cultivo\%20de\%20lupino\%20en\%20la\%20precordillera $\% 20 \mathrm{de} \% 20 \mathrm{la} \% 20$ VIII\%20region.pdf

Swanson, E., Harrgesel, M., Arnoldo, M., Sippell, M., Wong, R., 1989. Microspore mutagenesis and selection: canola plants with field resistance to imadozlinone. Theoretical and Applied Genetics 78, 525-530

Tan, S., Evans, R., Dahmer, M., Singh, B., Shaner, D., 2005. Imidazolinone-tolerant crops: history, current status and future. Pest Management Science 61, 246-257.

Tay, J., 2009. Producción de canola, lupino y arveja en la precordillera del Bío Bío y el secano costero de la provincia de Arauco. Boletín $\mathrm{N}^{\circ}$ 188. Instituto de Investigaciones Agropecuarias (INIA), Chillán, Chile. 166p. http://www2. inia.cl/medios/biblioteca/boletines/NR36469.pdf.

Timmis, J., Ayliffe, M., Huang, C., Martin, W., 2004. Endosymbiotic gene transfer: organelle genomes forge eukaryotic chromosomes. Nature Reviews Genetics 5(2), 123-135.

Tranel, P., Wright, T., 2002. Resistance of weeds to ALS inhibiting herbicides: what have we learned? Weed Science
50, 700-712.

Uchino, A., Watanabe, H., 2002. Mutations in the acetolactate synthase genes of sulfonylurea resistant biotypes of Lindernia spp. Weed Biology and Management 2(2), 104-109.

Uchino, A., Ogata, S., Kohara, H., Yoshida, S., Yoshioka, T., Watanabe, H., 2007. Molecular basis of diverse responses to acetolactate synthaseinhibiting herbicides in sulfonylurea-resistant biotypes of Schoenoplectus juncoides. Weed Biology and Management 7(2), 89-96.

Villarroya, M., Escorial, M., Garcia-Baudin, J., Chueca, M., 2000. Inheritance of tolerance to metribuzin in durum wheat. Weed Research 40, 293-300.

Walter, K., Strachan, S., Ferry, N., Albert, H., Castlea, L., Sebastian, S., 2014. Molecular and Phenotypic Characterization of Als1 and Als2 Mutations Conferring Tolerance to ALS Herbicides in Soybean. Pest Management Science 70(12), 1831-1839.

Wendel, J., 2000. Genome evolution in polyploids. Plant Molecular Biology 42, 225-249.

White, A., Graham, M., Owen, M., 2003. Isolation of acetolactate synthase homologs in common sunflower. Weed Science 51, 845-853.

Yang, H., Tao, Y., Zheng, Z., Zhang, Q., Zhou, G., Sweetingham, M., Howieson, J., Li, C., 2013. Draft genome sequence, and a sequence-defined genetic linkage map of the legume crop species Lupinus angustifolius L. PLOS ONE 8(5), e64799.

Yates de Saint-Pierre, F., 1993. Selectividad de herbicidas post emergentes en diferentes cultivares de lupino. Tesis Ingeniería Agronómica. Universidad Austral de Chile. 93 p. 